\title{
Metaplastic meningioma with pure and extensive cartilaginous transformation: A diagnostic dilemma*
}

\author{
Oumar Coulibaly ${ }^{1 \#}$, Justin Onen ${ }^{1}$, Amal Harmouch ${ }^{2}$, Boutarbouh Majhouba ${ }^{1}$, Adil Melhaoui $^{1}$, \\ Yasser Arkha ${ }^{1}$, Loubna Rifi ${ }^{1}$, Said Derraz ${ }^{1}$, Sanaa Sefiani ${ }^{2}$, Abdessamad El Ouahabi ${ }^{1}$, \\ Abdeslam El Khamlichi ${ }^{1}$
}

\footnotetext{
${ }^{1}$ Department of Neurosurgery, Hôpital des Spécialités, CHU Ibn Sina, Rabat-Salé, Morocco;

${ }^{\#}$ Corresponding Author: coulibalynch1@gmail.com

${ }^{2}$ Department of Pathology, Hôpital des Spécialités, CHU Ibn Sina, Rabat-Salé, Morocco
}

Received 17 July 2013; revised 20 August 2013; accepted 5 September 2013

Copyright (C) 2013 Oumar Coulibaly et al. This is an open access article distributed under the Creative Commons Attribution License, which permits unrestricted use, distribution, and reproduction in any medium, provided the original work is properly cited.

\begin{abstract}
Meningiomas are the most common extra-axial central nervous system tumours and often discovered in the middle to late adult life and especially in women. About $85 \%-90 \%$ of meningiomas are benign, $5 \%-10 \%$ are intermediate-grade, and $3 \%-5 \%$ are malignant. Metaplastic meningioma is a rare subtype of WHO Grade I meningioma histologically characterized by the presence of mesenchymal components. The presence of pure and extensive cartilaginous differentiation in meningiomas is extremely rare and remains a diagnostic dilemma. We report, perhaps the first case of this entity in a 52-year-old woman and discuss the pathogenesis, the imaging features and the histopathologicals data.
\end{abstract}

Keywords: Meningioma; Metaplastic Meningioma; Pure Cartilaginous Differentiation;

Diagnostic Dilemma

\section{INTRODUCTION}

Meningiomas are the most extra-axial central nervous system tumours and often discovered in the middle to late adult life, especially in women, and account approximately $15 \%-30 \%$ of all intracranial tumors [1]. They are classified into three groups according to the World Health Organisation (WHO) grading system based upon morphologic criteria with characteristic pathologic and imaging features: benign meningiomas in $85 \%-90 \%$ (WHO Grade I), atypical meningiomas in 5\% - 10\%

"Disclosure: The authors have no personal financial or institutional interest in any of the drugs, materials, or devices described in this article.
(WHO Grade II) and malignant meningiomas in 3\% - 5\% (WHO Grade III) [2]. Considering this grading, most of symptomatic patients, in whom the diagnosis of meningiomas is suggestive, undergo a surgical resection to relieve neurological symptoms and often allow us to guide therapeutic implications after histologic data. But sometimes, the diagnosis remains difficult despite all these efforts. Metaplastic meningioma is a rare subtype of WHO Grade I meningioma histologically characterized by the presence of mesenchymal components, including osseous, cartilaginous, lipomatous, myxoid or xanthomatous and cartilaginous tissue [3]. But, the presence of pure and extensive cartilaginous differentiation in this entity of meningiomas is very uncommon. We discuss herein the diagnostic dilemma in this kind of meningioma.

\section{CASE REPORT}

A 52-year-old woman, followed for diabetes type II on nutritional regime, had been admitted in our department for a 10-year history of isolated headaches for which she opted for self medication by analgesics. Due to persistence of the headaches and despite analgesic therapy, she sought medical help in our department to ascertain the probable underlying cause. On admission, neurological examination was strictly normal (no deficit and no papilledema on ophthalmological exam). A differed brain CT scan was done and revealed a left parietal spontaneously hyperdense and heterogeneous lesion with no significant modification following contrast injection and suggestive of dural and bone infiltrations (Figure 1). Cerebral MRI to refine the radiologic diagnosis complemented these data. Axial, sagittal and coronal contrast enhanced T1W MRI showed the same heterogeneous lesion not much modified by Gadolinium injection with probable dural and bone thickening and without brain edema or midline shift (Figure 2). Our initial diagnosis 


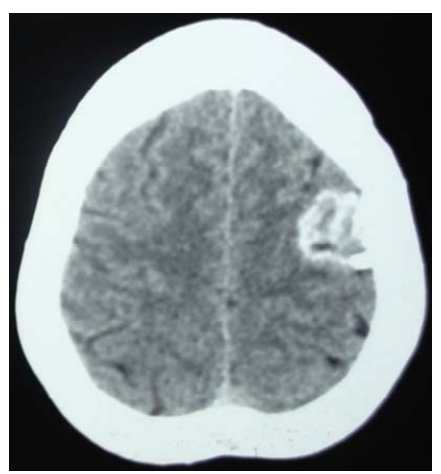

(a)

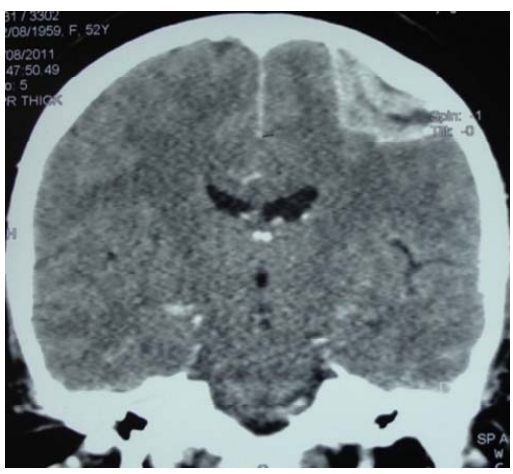

(b)

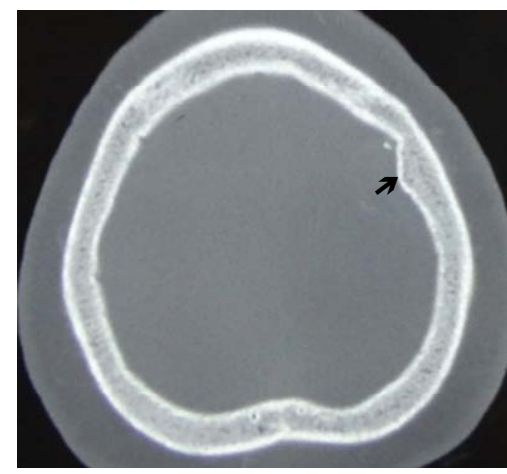

(c)

Figure 1. CT scan views showed a left parietal spontaneous hyperdense and heterogeneous lesion (a) with no significant much modification following contrast injection (b) and suggesting of dura and bone infiltrations (c).

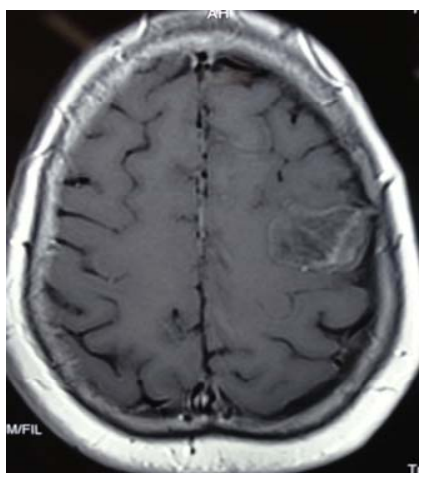

(a)

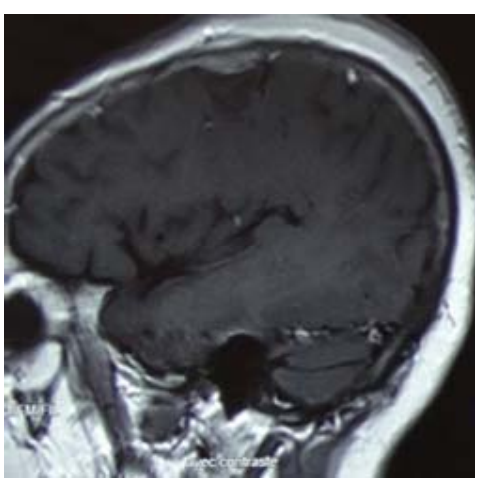

(b)

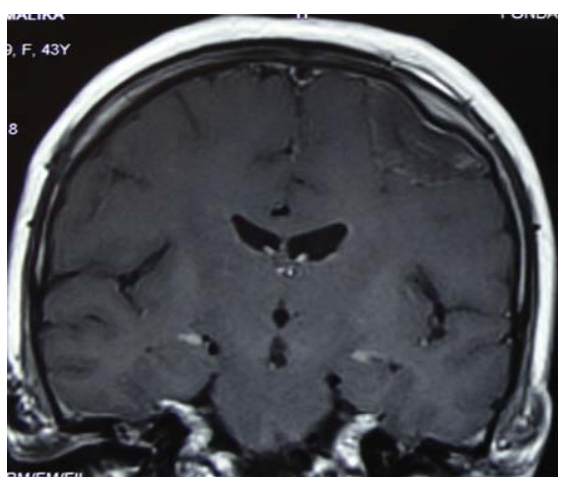

(c)

Figure 2. Axial (a), sagittal (b) and coronal (c) contrast enhanced T1W MRI showed a left parietal heterogeneous lesion not much modified by Gadolinium injection with probable dural and bone thickening and without brain edema or parenchyma shift.

basing on the radiological appearances was parietal convexity meningioma or intracranial tuberculoma. She underwent a left craniotomy centred on the lesion that permitted an "en bloc" resection of this tumour together with the overlying dura (Simpson's Grade I). Per operatively, the lesion was yellowish, firm and totally hard, with a very good plain of cleavage, and a very loose dural attachment, leaving a cavity that was in conformity to the shape of the lesion and measuring $4.5 \mathrm{~cm} \times 2.5 \mathrm{~cm} \times$ $1.5 \mathrm{~cm}$ (Figure 3). Extemporaneous histopathological examination was in favour of a cartilaginous tumor. These histological data were accepted with difficulty by our team and we recommended extensive studies in order to properly confirm the diagnosis. Finally, the diagnosis of metaplastic meningioma with extensive cartilaginous transformation was made after HES staining (Figure 4). Postoperative CT and MRI scans revealed no residual tumour (Figure 5). She was discharged from hospital without neurological signs 08 days after surgery and is symptom-free 24 months following surgery.

\section{DISCUSSION}

Tumors derived from "meningeal cells" were described in the older medical literature on several names, depending on their source and published as psammoma, dural sarcoma, dural endothelioma, fibrosarcoma, angioendothelioma, endotheliosis of the meninges, meningeal fibroblastoma, meningoblastoma, mestothelioma of the meninges, and others [4,5]. But in 1922, Harvey Cushing (1869-1939) had been the first to propose the term of "meningioma" to describe these tumors and to end of to a series of appointments without end [4]. These meningiomas are the most common primary extra-axial intracranial tumors and represent one-third of all such tumors [6]. They exhibited a wide spectrum of microscopic appearance and capacity for mimicking the histological features of other neoplasms [7]. They are almost predominantly benign, slow growing lesions and constitute $15 \%-30 \%$ of all intracranial tumors [8]. They occur two or three times more commonly in women between 40 and 70 years old. Regarding their capability to express both mesenchymal and epithelial characteristics, the 1993 WHO classification of the tumors of the meninges recognized some histologic variants of meningiomas from which a metaplastic meningioma was listed [9]. The current WHO histopathological classification deter- 


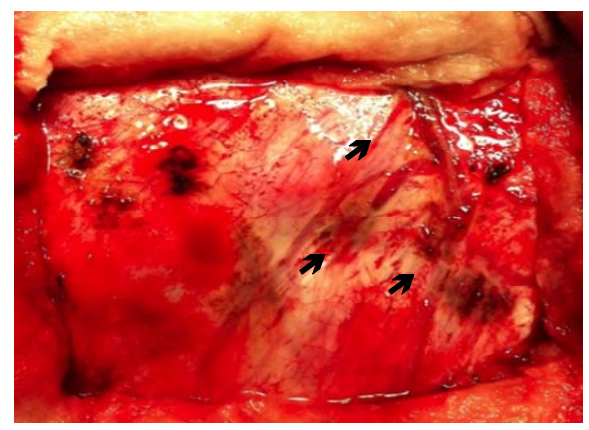

(a)

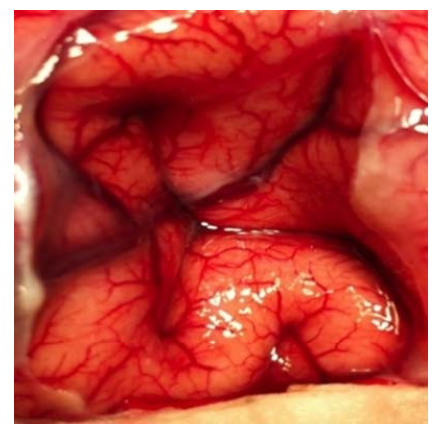

(b)

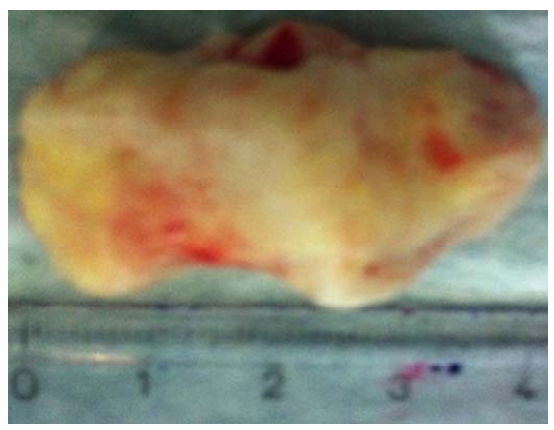

(c)

Figure 3. Operative view showing dimpling at the dural surface (a), parenchymal remodelling conforming to the tumour shape (b), removed tumour specimen (c).

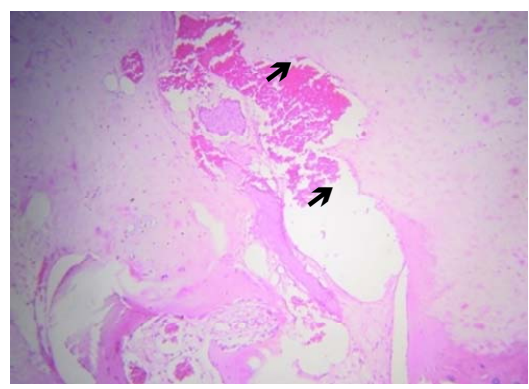

(a)

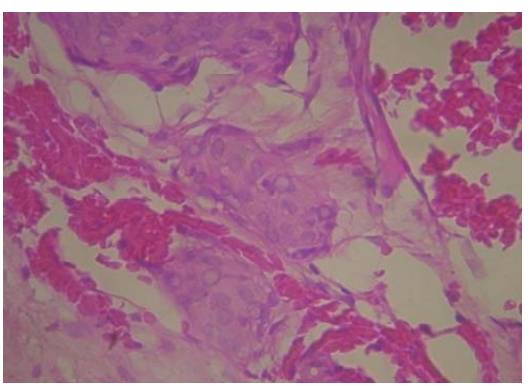

(b)

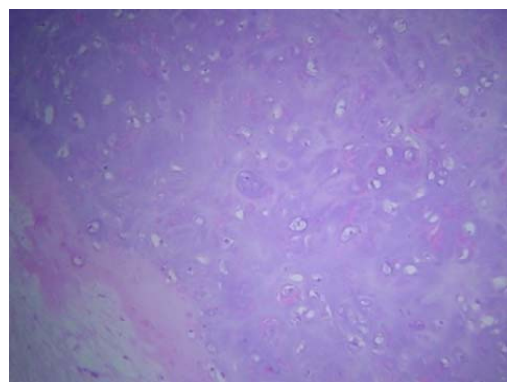

(c)

Figure 4. (a) HES X 100, chondroid pure tissue sheltering (surrounding) arachnoid proliferation (see arrows); (b) HES X 400, arachnoid cells with pseudo inclusions wound in clusters; (c) HES X 100, regular and pure chondroid tissue.

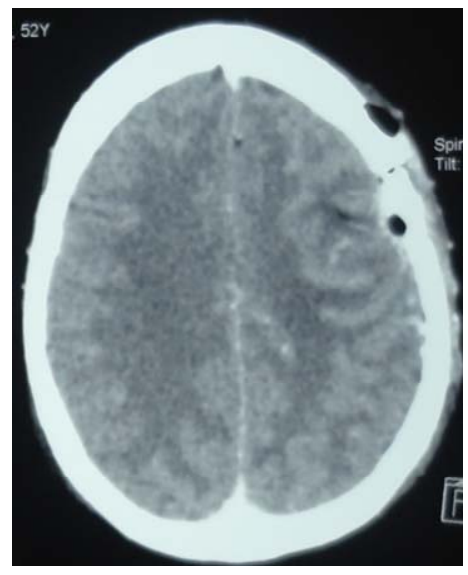

(a)

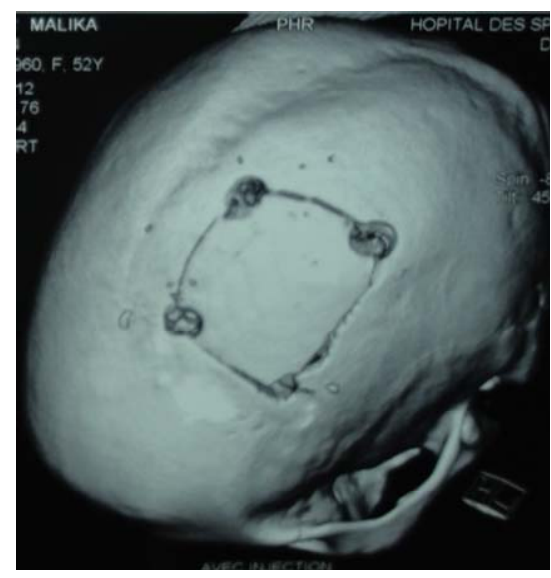

(b)

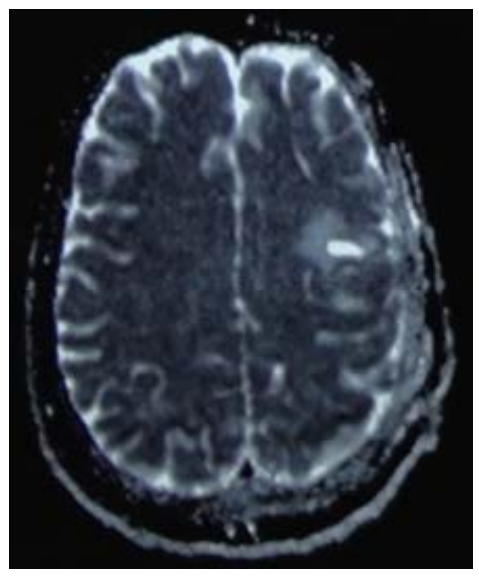

(c)

Figure 5. Postoperative CT scan showing no residual tumour (a); bone-flap on bone window (b); Postoperative MRI view showing no residual tumour $(\mathrm{c})$.

mines 15 separate histopathological variants of meningiomas that correspond to 3 grades of malignancy: typical or benign meningiomas (Grade I), atypical meningiomas (Grade II) or malignant meningiomas (Grades III and IV) [10,11]. This subtype of meningiomas called "metaplastic meningioma" and rated as WHO Grade I meningioma, refer to a group of tumors marked histologically by the presence of focal mesenchymal differentiations or components, including osseous, cartilag- inous, lipomatous, myxoid or xanthomatous tissue [3,11]. These morphological and histological changes in meningiomas pose therapeutic challenges to pathologists, neurosurgeons and oncologists; this is why it was useful to have a prompt diagnosis before therapy in this group in order to keep their aggressiveness. Becker C. et al. in 1999 describe a case of metaplastic meningioma from which it was noted large areas of a typical meningothelial meningioma with numerous cartilaginous islands and 
some chondroid regions [12]. Metaplastic meningioma with pure and extensive cartilaginous transformation is very uncommon. In our knowledge and basing on the literature research, any case like ours had been published before.

The mechanism of cartilaginous transformation in these meningiomas is still unclear and debated. However, some hypotheses about the pathogenesis of these metaplasias should be discussed. Willis R.A. in 1967 [13] argued that meningocytes are mesenchymal in origin, multipotent mesenchymal cells have the ability to differentiate into fibrous, mucoid, adipose, synovial, meningeal, cartilaginous, osseous, hematopoietic, vascular, or reticuloendothelial tissues and may inappropriately respond to an unknown stimulus (precursor) to produce a metaplastic meningioma. Awadesh, et al. in 2011 [14] said that metaplasia is a reversible change in which one adult cell type is replaced by another adult cell type. This may represent an adaptive substitution of cells. This implies that arachnoidal cap cells, from which meningiomas arise, may undergo gradual transformation into other cell types such as fat, bone, cartilage and myxoid tissue. But this variant differentiation has no prognostic significance [15]. Despite all these explanations, we are not able to confirm why these metaplastic changes occur in some meningiomas and why not in others. Jing Xiang Hung et al. in 2011 also said, to date it is not known how they occur too [16]. The clinical features found in our patient are similar to other clinical course of meningioma in this location, but the radiological finding could not precise the diagnosis prior the operation. This is why, the authors thought to others differentials diagnosis before surgery. Our patient was successfully operated with gross total removal. These data are supported by Ulivieri S. et al. (2008) who argued, the imaging data and a surgical strategy had to be always valued and used [17]. Histologically in our case, there was essentially a pure and regular cartilaginous tumor proliferation with very focally small clusters of meningeal cells rounded nucleus containing intranuclear inclusions and clear cytoplasm scanty. Becker et al. (1999) [12] also described a typical meningothelial meningioma from which numerous cartilaginous islands and some chondroid regions, obviously of intermediate (meningothelial/cartilaginous) differentiation could be seen. However, Jing X. H. et al. [16] described a case of an intracerebral metaplastic meningioma with prominent ossification and extensive calcification. Meningiomas with a pattern corresponding to at least rare two histological variants (secretory and lipomatous components or chordoma-like and chondromalike structures) have been sporadically reported $[18,19]$. In our knowledge, our case is the unique published metaplastic meningioma to be a purely and extensive cartilaginous differentiation. These extensive cartilaginous metaplasias are very unusual but have similar prognosis behaviour as the other subtypes in Group I. Unfortunately, true that "tumors from meningeal cells" called meningiomas now had been outlined since the sixteenth century; but they continue to be a challenging environment in the neurosurgeon's daily activities especially with these constant changes and very low risk of recurrence or aggressive growth. Such research is still pending to unravel this mystery.

\section{CONCLUSION}

Metaplastic transformation in meningiomas may follow a long-standing tumour (meningioma) development, which denotes a change from arachnoid to mesenchymal components. How much time these metaplastic changes take? After this transformation, it is not clearly known the state of events that follow. As was the case with our patient, it is possible that the lesion remained stable thereafter. These lesions pose a diagnostic dilemma on standard brain imaging techniques. Till presently, there is no clear-cut therapeutic protocol, but proponents for surgical strategy abound. Therefore, we do feel that future research is required to unravel the mystery that surrounds this particular type of metaplasia and whether it does harbour the possibility of evolution. For the moment, radical resection remains the rule.

\section{ACKNOWLEDGEMENTS}

We would like to thank Mrs. Barry Najah for her supports for this manuscript.

\section{REFERENCES}

[1] Er, U., Gürkanlar, D., Kazanci, A., Şimşek, S. and Bavbek, M. (2006) Lipomatous meningioma: Case report and diagnostic pitfall. Turkish Neurosurgery, 16, 40-43.

[2] Lynch, J.C., Ferreira, L.A., Welling, L. and Schulz, R.C. (2008) Multiple intracranial meningiomas: Diagnosis, biological behavior and treatment. Arquivos de Neuro-Psiquiatria, 66, 702-707. http://dx.doi.org/10.1590/S0004-282X2008000500018

[3] Louis, D.N., Ohgaki, H., Wiestler, O.D., et al. (2007) The 2007 WHO classification of tumours of the central nervous system. Acta Neuropathologica, 114, 97-109. http://dx.doi.org/10.1007/s00401-007-0243-4

[4] Cushing, H. (1922) The meningiomas (dural endotheliomas): Their source, and favoured seats of origin. Brain, 45, 282-316. http://dx.doi.org/10.1093/brain/45.2.282

[5] Cushing, H.L. and Eisenhardt, L. (1938) Meningiomas: Their classification, regional behavior, life history and surgical end results. Charles C. Thomas, Springfield.

[6] Wiemels, J., Wrensch, M. and Claus, E.B. (2010) Epidemiology and etiology of meningioma. Journal of NeuroOncology, 99, 307-314. 
http://dx.doi.org/10.1007/s11060-010-0386-3

[7] Kepes, J.J. (1986) Presidential address: The histopathology of meningiomas. A reflection of origins and expected behavior? Journal of Neuropathology \& Experimental Neurology, 45, 95-107. http://dx.doi.org/10.1097/00005072-198603000-00001

[8] Louis, D.N., Ohgaki, H., Wiestler, O.D. and Cavenee, W.K. (2007) WHO classification of tumours of the central nervous system. 4th Edition, International Agency for Research on Cancer, Lyon.

[9] Lopes, M.B.S., VandenBerg, S.R. and Scheithauer, B.W. (1993) The World Health Organization classification of nervous system tumors in experimental neuro-oncology. In: Levine, A.J. and Schmidek, H.H., Eds. Molecular Genetics of Nervous System Tumors, Wiley-Liss, New York, 1-36.

[10] Mahmood, A., Caccamo, D.V. and Tomecek, F.J. (1993) Atypical and malignant meningiomas. A clinicopathological review. Neurosurgery, 33, 955-963. http://dx.doi.org/10.1227/00006123-199312000-00001

[11] Kleihues, P. and Cavenee, W.K. (World Health Organization) (2000) Classification of tumours. Pathology \& genetics. Tumours of the nervous system. IARC Press, Lyon, 175-192.

[12] Becker, C., Kuchelmeister, K., Richter, H.P. and Schachenmayr, W. (1999) Metaplastic meningioma with cartilagenous differentiation. Pathologe, 20, 335-339. http://dx.doi.org/10.1007/s002920050367

[13] Willis, R.A. (1967) Pathology of tumors. 4th Edition, Butterworths, London, 658-662, 736-746.

[14] Jaiswal, A.K., Mehrotra, A., Kumar, B., Jaiswa, S., Vij, M., Behari, S. and Pal, L. (2011) Lipomatous meningioma: A study of five cases with brief review of the literature. Neurology India, 59, 87-91.

[15] Roncaroli, F., Scheithauer, B.W., Laeng, R.H., Cenacchi, G., Aleff, P.A. and Moschopulos, M. (2001) Lipomatous meningioma. A clinicopathologic study of 18 cases with special reference to the issue of metaplasia. The American Journal of Pathology, 25, 769-775. http://dx.doi.org/10.1097/00000478-200106000-00008

[16] Huang, J.X. and Peterson, F. (2011) Intracerebral metaplastic meningioma with prominent ossification and extensive calcification. Rare Tumors, 3, e20.

[17] Ulivieri, S. and Oliveri, G. (2008) Lipidised faux meningioma: Case report. AJNS, 27.

[18] Matyja, E., Nagańska, E., Zabek, M. and Jagielski, J. (2005) Meningioma with the unique coexistence of secretory and lipomatous components: A case report with immunohistochemical and ultrastructural study. Clinical Neuropathology, 6, 257-261.

[19] Matyja, E., Grajkowska, W., Lazarczyk, M., Marchel, A. and Czernicki, T. (2006) Chordoid meningiomas of a different histopathological pattern: A report of two cases. Folia Neuropathologica, 44, 34-41. 